KPIs mit an die Hand. Mit Vorträgen zu „Compliance“, „Kommunikation im Projektmanagement“ und „Führungskräftemangel“ wurden anschließend weitere Themen diskutiert, denen sich Controller in ihrer Rolle als Business Partner stellen sollten. Beschlossen wurde die Veranstaltung mit einem Vortrag von Karl-Heinz Steinke, Mitglied des Vorstands des ICVs, zu „Controlling Excellence“. Er zeigte die Treiber erfolgreichen Controllings auf. „Technische Excellence ist nur notwendige Voraussetzung“, betonte Steinke. Wesentlich für den Erfolg von Controllern seien die Kom- munikation und die Adaption. Controller sollten ihr eigenes Controllerbild regelmäßig anpassen, um ihren Rollenwert in einem Unternehmen nicht zu verlieren.

Die Teilnehmer waren sich am Ende einig: Controller werden in ihren Unternehmen zukünftig verstärkt über den Tellerrand schauen. Damit sie nicht den Anschluss verlieren und sich mit Kollegen austauschen können, bedarf es auch Veranstaltungen wie der CAB.

Vera Treitschke, Wiesbaden

\title{
Der Controller als Kreativgeist und Navigator
}

\section{CIS Controlling Insights Steyr, Oberösterreich}

Der globale Wettbewerb wird härter, die Märkte werden zusehends volatiler. Darauf müssen sich Controller einstellen. Bei der Fachtagung Controlling Insights in Steyr lautete das Thema dementsprechend „Triple-A Controlling: Unternehmenserfolg im globalen Wettbewerb“. Bei einer Podiumsdiskussion wurden Vergleiche gezogen, wie sich die Arbeit eines Controllers in den vergangenen fünf Jahren seit der LehmanPleite verändert hat und welche Lehren für die Unternehmenssteuerung von morgen daraus gezogen werden können. „Wir waren 2008 von der Heftigkeit des Einbruchs völlig überrascht“, sagte Peter Köstler, Vizepräsident des Automobilzulieferers Miba mit Zentrale im oberösterreichischen Laakirchen. Man sei von Umsatzrückgängen von fünf bis zehn Prozent ausgegangen. Am Ende seien es dann bis zu 55 Prozent gewesen. Als Folge daraus arbeiten Controller beim MibaKonzern heute viel mehr mit Szenarien. Es stünden heute weit mehr Instrumentarien zur Verfügung als 2008. Das Risikomanagement spiele eine größere Rolle. Rudolf Peterbauer, Controlling-Leiter des Batterie-Produzenten Banner, warf ein, dass Controller heute mehr die Funktion eines Kreativgeistes ausüben. Initiative sei gefragt. „Früher hatte der Controller den Ruf des Zahlenpapstes. Später war er auch Kommunikator. Heute treibt er die Dinge voran. “ Für Christoph Wurm, Vorstandsdirektor der VKB-Bank, sind Controller in den Banken die Navigatoren, die es in stürmischen Zeiten braucht. Neben der Podiumsdiskussion lauschten die Teilnehmer den Referaten der Vortragenden, die in diesem Jahr vorwiegend aus der Automobil-Branche stammten. Günter Apfalter,
Präsident des internationalen Automobilzulieferers Magna Steyr und Präsident von Magna International Europe, sprach über Wettbewerbsfähigkeit und Innovation als Treiber nachhaltiger Standortabsicherung. Das Wachstum spiele sich nicht in Europa, sondern in Asien ab. Um konkurrenzfähig zu bleiben, fordert Apfalter mehr Flexibilität ein, so z. B. Arbeitszeitkonten. Der Manager kritisierte, dass Unternehmen in Österreich bis zu 60 Prozent weniger in Forschung und Entwicklung investieren als in Deutschland. Johann Habring, Vorstandsmitglied der IFN Holding, berichtete ausführlich über den Bereich Planung im Controlling. Auch in einem Konzern soll die Planung laut Habring dezentral ablaufen. Planung sei abhängig von Struktur, Größe und Organisation eines Unternehmens. Das System eines Unternehmens lasse sich nicht einfach für andere übernehmen. Die dezentrale Verantwortung der Abteilungsleiter für die Planungen müsse von Controllern eingefordert werden. „Der Plan ist nicht der des Controllers, sondern des Unternehmens und seiner Bereiche. "Martha Oberndorfer, Vorstand der Österreichischen Bundesfinanzierungsagentur, rundete die Tagung ab, indem sie strategische Aspekte eines erfolgreichen Staatsschuldenmanagements am Beispiel der Republik Österreich aufzeigte.

Viele der 350 Teilnehmer in Steyr sind Stammgäste. Sie werden sich beim 13. CIS Controlling Insights Steyr im November 2014 wiedersehen.

Stefan Minichberger, Steyr 